\title{
Controle Et Performance Des Reseaux De Pme Camerounaises : Une Approche Transactionnelle
}

\author{
Tcheubeu Nana Tyéri B. \\ Ngomesse Njike Désirée
}

PhD Enseignant chercheur Université de Dschang (IUTFV-Bandjoun)

doi: 10.19044/esj.2017.v13n7p215 URL:http://dx.doi.org/10.19044/esj.2017.v13n7p215

\begin{abstract}
Nowadays, companies, and especially SMEs, are engaged in fierce competition. One of the solutions to this strong competition is for these companies to reduce the number of potential suppliers and to establish strategic partnership relationships. This is how a networked organization allows them to be more flexible and better able to adapt to this environment. Research, however, reveals a very weak life experience of business networks. The objectives of this work are to present the control in the transactional approach within SME networks, to check whether it can explain the performance of these SMEs. From an observation of 54 Cameroonian SMEs in the manufacturing and logistics sectors, it is clear that among the tools of the transactional control mode, contracts less than six months are the most used and these negatively influence the performance of network SMEs. This important use of contracts of less than six months may be due to non-compliance with the terms of the contract by the partners, legal and judicial insecurity which leads the partners to evaluate first the performance of a contract less than six months, before moving on to another contract.
\end{abstract}

Keywords: SME network, control in transactional theory, performance of SME networks

\section{Résumé}

De nos jours, les entreprises, et particulièrement les PME se livrent à une concurrence acharnée. L'une des solutions à cette forte concurrence consiste pour ces entreprises à réduire le nombre de fournisseurs potentiel et à nouer des relations stratégiques de partenariat. C'est ainsi qu'une organisation en réseau leur permet d'être plus flexible et de mieux s'adapter à cet environnement. Les recherches relèvent cependant une expérience de vie très faible des réseaux d'entreprises. Les objectifs de ce travail sont les 
suivants : présenter le contrôle dans l'approche transactionnelle au sein des réseaux de PME, vérifier si celui-ci peut expliquer la performance de ces PME. A partir d'une observation auprès de 54 PME camerounaises des secteurs manufacturiers et logistiques, il ressort que, parmi les outils du mode de contrôle transactionnel, les contrats d'une durée de moins de six mois sont les plus utilisés et ceux-ci influencent négativement la performance des PME en réseau. Ce recourt important aux contrats de moins de six mois peut être dû au non respect des termes du contrat par les partenaires, à l'insécurité légale et judiciaire qui amène les partenaires à évaluer d'abord l'exécution d'un contrat de moins de six mois, avant de passer à un autre contrat.

Mots clés: Réseau de PME, contrôle dans la théorie transactionnelle, performance des réseaux de PME

Il devient difficile depuis quelques années de prévoir le développement des PME sans s'interroger sur leur capacité à s'adapter à la mondialisation de l'économie. En effet, cette mondialisation engendre de nombreux changements dans le macro et dans le micro-environnement des PME, perturbant leur position sur le marché. Elle détermine, par exemple, les baisses de prix et formule des exigences plus élevées en matière de technologies, de qualité, de diversité et d'innovations des produits et services. En renforçant la concurrence nationale et internationale, elle modifie aussi les relations traditionnelles établies entre les PME et les autres acteurs économiques (fournisseurs, distributeurs, etc.), tout comme elle intensifie la confrontation des PME locales avec de grandes entreprises multinationales et des concurrents particulièrement compétitifs (Crottet, 2001).

A cet effet, la ruée vers l'innovation est forte. Pour y faire face les entreprises développent des nouvelles formes organisationnelles: les coopérations ${ }^{46}$ qui peuvent prendre plusieurs formes (partenariat, joint venture, alliance, entreprise en réseau, réseau d'entreprise, entreprise virtuelle, entreprise en grappe, etc.). Pour ces formes, l'entreprise n'a plus de frontière aussi nette, elle s'etend vers ses clients, fournisseurs, et concurrents etc. La PME face à ces mutations, n'est plus en mesure de tout faire en interne ; elle tente de mettre ses forces en valeur et de s'orienter vers ses partenaires en procédant à des efforts de rationalisation. Elle va se concentrer sur ses métiers de base et faire appel à des partenaires efficaces pour ses activités complémentaires et ce, dans une recherche de flexibilité, de

46 La coopération semble être l'outil privilégié pour faire face à l'instabilité de l'environnement. 
variabilité, de l'utilisation optimale des équipements, et de compétitivité susceptible de consolider sa position sur le marché (Hammami et al, 2001). Les réseaux d'entreprises enregistrent un développement croissant ${ }^{47}$, mais la complexité de leurs organisations et de leurs gestions entraine un taux de mortalité élevé, entre 37 et $70 \%$ selon les secteurs. Les études de Porter (1986) sur l'espérance de vie de ces nouvelles formes d'entreprises est de 4,7 ans aux Etats Unis, et en 1998 le même auteur montre que moins de 40 \% atteignent 4 ans et moins de $15 \%$ atteignent 10 ans. Par ailleurs, ces partenariats demeurent souvent fragiles et sujets à une grande instabilité, avec un taux d’échec élevé atteignant les 90 \% (Harrigan 1985; Porter, 1986). «Plusieurs entreprises camerounaises optent actuellement pour l'externalisation » ${ }^{48}$. Si l'externalisation présente un transfert d'activités vers un prestataire, cette situation suppose de gérer le cœur de métier de l'entreprise (Quélin 2003). A cet effet et à titre d'exemple, l'Aéroport de Paris a externalisé la majorité de ses activités et cette entreprise occupe une meilleure position concurrentielle dans son secteur d'activité. Pour que le réseau fonctionne normalement, il faut que chaque partenaire trouve un intérêt ou encore atteint ses objectifs et ait satisfaction. Pour y parvenir, il revient à chaque partenaire de mettre sur pied les outils et les techniques de management, plus particulièrement de contrôle pour permettre de se rassurer de l'atteinte de ses objectifs. Selon Gulati (1998), la recherche est foisonnante en ce qui concerne les causes de la formation du réseau ou de sa structure, ce n'est pas le cas pour la mise en œuvre et la gestion qui restent peu étudiés. Plusieurs auteurs ont soulignés le faible volume des travaux touchant à la thématique de coordination des réseaux inter organisationnels et notamment le contrôle (Hasrouri, 2007). Au sein d'un réseau, les mécanismes de contrôle se manifestent dès la sélection des partenaires, le suivi pendant le déroulement de la relation qui peut aller d'un contrôle faible avec des relations fondées sur la confiance et une compréhension mutuelle, à un contrôle total utilisant des procédures très bureaucratiques. Deux types de régulation y sont souvent analysés : celui fondé sur le contrat et celui reposant sur la confiance. La notion de contrat renvoie à la fois aux outils juridiques utilisés pour organiser le réseau et aux aspects formels de la coordination entre partenaires (Roblédo, 1997). La notion de confiance, malgré le vif intérêt des chercheurs en sciences de gestion (Guibert, 1999 ; Gurviez et Korchia, 2002), est paradoxalement plus difficile à circonscrire.

\footnotetext{
${ }^{47}$ Selon les statistiques fournies par (HARRIGAN K. R., 1988 : p. 145) « 1393 accords de coopération sont crées aux Etats-Unis entre 1978 et 1986 contre seulement 380 pour toute la période antérieure "

${ }^{48}$ Plus de 78,9\% de l'échantillon de NGOK EVINA (2008) nous donne ces chiffres après une étude auprès des PME camerounaises.
} 
Nous nous intéresserons dans le cadre de cette recherche au contrôle formel ou encore l'approche transactionnelle.

Le contrôle permet de mesurer la performance. Les rôles assignés au contrôle organisationnel restent le pilotage de la performance et le pilotage du changement. L’idée de la performance est inscrite dans la logique : objectif-moyen-résultat. Ce qui renvoie aux notions d'efficacité et d'efficience. Le pilotage du changement consiste à maîtriser les facteurs clés de succès et les équilibres financiers pour atteindre les objectifs fixés. Les éléments qui permettent de définir la performance des réseaux d'entreprises sont jusqu'ici trop variables d'un contexte à l'autre et mal connus. Cette mauvaise connaissance se traduit par le fort taux de mortalité de ces entreprises en coopération. Ceci nous amène à poser la question suivante : est ce que le contrôle formel permet d'avoir une influence sur la performance des réseaux de PME camerounaises ? A notre connaissance aucun travail ne s'est intéressé à cette problématique. Les objectifs de ce travail sont les suivants: présenter le contrôle formel au sein des réseaux de PME camerounaises, vérifier si ce contrôle formel peut expliquer la performance de ces PME. Notre unité d'analyse est la PME qui est constituée de la très petite entreprise, de la petite entreprise, et la moyenne entreprise ${ }^{49}$; Pour atteindre ces objectifs nous ferrons d'abord une revue de la littérature sur l'approche transactionnelle du contrôle (contrôle formel), ensuite sur la performance des entreprises en réseaux, enfin nous verrons s’il y a un lien entre le contrôle formel et la performance.

\section{Approche théorique du controle formel et la performance des réseaux de pme}

Le réseau inter organisationnel peut être défini comme une relation à long terme entre les organisations. Nous substituons ici le réseau à la coopération (le seul élément de distinction étant la durée de la relation), avec trois paramètres centraux : l'idée de coopération inscrite dans une stratégie volontaire ou contrainte, la nécessité d'intégrer à cette coopération des relations de long terme entre acteurs indépendants et le besoin de relations suivies et fournies. Selon Butera, (1991), "l'entreprise réseau est un ensemble d'entreprises liées les unes aux autres par un cycle de production. Le lien n'est ni juridique, ni structurel ; il revêt souvent la forme de simples accords. Ces entreprises ont en commun un puissant système de coopération fonctionnelle » p. 2. Cette définition s'appuie plus sur la filière de production, celle de Podolny et Page (1998) est plus élargie et considère le réseau d'entreprises comme " une collection d'entreprises qui poursuivent

${ }^{49}$ Loi $n^{\circ}$ 1010/001 du 13 avril 2010 portant promotion des PME au Cameroun dans ses articles 4,5 et 6 . 
des relations d'échanges répétées et durables les unes avec les autres et qui, en même temps, n'ont pas d'autorité organisationnelle légitime pour arbitrer et résoudre les disputes qui peuvent se produire pendant l'échange » p. 2. Pour nous, le réseau d'entreprises est constitué à partir de deux entreprises qui ont des relations depuis au moins deux ans, sous des formes juridiques diverses tout en restant juridiquement indépendantes afin de mener à bien un projet, pouvant aller du simple partage d'une ressource jusqu'à la réalisation d'une innovation en commun ${ }^{50}$.

\section{La théorie sur l'approche transactionnelle du contrôle dans les réseaux de PME}

Le succès d'une organisation est conditionné par les actions en interne aussi bien que par les actions d'un grand nombre d'acteurs externes. Le problème est alors de savoir comment organiser les relations avec ces acteurs externes et comment influencer ces partenaires qui ne répondent pas aux conditions hiérarchiques traditionnelles. Il s'agit de comprendre le contrôle inter organisationnel. La théorie des coûts de transactions développée par Williamson (1975) propose différents arrangements institutionnels pour gérer les transactions de manière efficiente. Toute transaction génère un coût qui vient s'ajouter au seul coût du produit échangé. C'est le coût de la recherche du partenaire, de négociations, de contractualisation ou les coûts de la structure qui contrôle le déroulement du contrat, des dispositifs de surveillance ou les coûts liés à une mauvaise adaptation du contrat ainsi que les coûts de sa renégociation éventuelle, et les coûts d'opportunité que représente l'immobilisation de certains actifs destinés à garantir le respect des clauses du contrat. Ce coût dépend des caractéristiques des transactions : leur fréquence, leur incertitude et le niveau d'actifs spécifiques qu'elles requièrent (exemple actifs matériels ou humains non redéployables dans le cadre d'un autre échange). Il est également influencé par les facteurs humains (opportunisme et rationalité limitée) et environnementaux (incertitude, nombre d'acteurs potentiels sur le marché) dans lesquels ont lieu les transactions. L'objectif de la théorie est de proposer le mode de gouvernance qui permet de minimiser le coût des transactions compte tenu de leurs caractéristiques et du contexte dans lequel elles se déroulent.

Selon cette théorie trois formes de gouvernances sont proposées : le marché, la forme hybride et la hiérarchie. Ces structures diffèrent dans les mécanismes de contrôle utilisés pour garantir la bonne exécution des

50 Il convient de distinguer les groupements dont nous parlons ici des groupements momentanés d'entreprises définis comme une agrégation temporaire de compétences et de ressources qui collaborent ensemble pour un besoin spécifique et limité dans le temps, telle une opportunité d’affaire. 
transactions. Dans la gouvernance par le marché, le contrôle provient de la concurrence libre et parfaite. Toute l'information est contenue dans le prix. Le contrat qui soutient cette forme est classique (complet et détaillé). La rupture d'une relation n'a pas de conséquences puisqu'une nouvelle relation peut commencer à tout moment dans les mêmes conditions. En phase de sélection: les partenaires sont choisis sur la base d'un appel d'offres concurrentiel concernant les résultats. Les partenaires potentiels sont nombreux étant donné que les actifs requis pour la transaction sont peu spécifiques. Le contrat classique fixe les règles de fonctionnement de la coopération. Ce contrat n’a pas besoin d’être détaillé. En effet, la possibilité qu'a chacun de mettre l'autre en concurrence à chaque instant lui donne l'assurance que son partenaire se disciplinera pour agir de manière efficace et efficiente. En phase d'exécution: le contrôle par le marché s’organise autour d'un processus. Il passe par la mise en place d'un mécanisme ex ante : fixation des objectifs, élaboration de la structure d'incitation. Il comporte également des dispositifs ex post : évaluation des performances et mise en œuvre des sanctions et des récompenses qui se traduisent souvent par la reconduite ou non du contrat.

Dans la gouvernance hybride, le contrôle provient du contrat néoclassique dans lequel chaque partie accepte l'assistance d'un tiers (un arbitre) dans la résolution des conflits et l'évaluation de l'exécution. D'autres mécanismes de sauvegarde (exemple des otages) permettent de renforcer sa non-remplaçabilité ou, tout au moins, d'augmenter les coûts de changement de partenaires. Le contrôle peut être défini autour de deux pôles principaux : (1) l'évaluation, le suivi et l'audit supervision directe, partage des informations, communication approfondie et fréquente, comportement ; (2) le contrat de partenariat. Le contrat est généralement considéré comme l'élément central des procédures de contrôle formel des partenaires. Ainsi que le démontre Rouette (1999), l'importance des différentes composantes du contrôle varie selon le stade du cycle de vie du réseau. La formalisation du contrat a généralement lieu lors des phases de négociations et d'ententes et a pour objet la définition des conditions générales de coopérations ainsi que les obligations de chacune des parties. Le degré de complexité du contrat varie selon le nombre et la nature des obligations considérées (obligations en termes de résultats, de performance, de contrôle, contraintes en termes de pénalités et de responsabilités).

Dans la gouvernance hiérarchique, le contrôle provient de dispositifs contractuels personnalisés pour lesquels le système de sanction/récompense est à long terme ainsi que des mécanismes hiérarchiques à l'instar de l'autorité (Nogatchewsky, 2009). Il repose sur les mécanismes que l'on trouve dans la structure hiérarchique : des règles, standards et procédures spécifiques. En phase de sélection : les partenaires sont choisis sur la base de 
critères détaillés au cours d'appels d'offres formels. Les compétences sont spécifiquement prises en compte par ces critères. Le contrat qui scelle la coopération est très détaillé. Il y inclut les conséquences des contingences futures sur le déroulement des échanges. En cas de désaccord entre les parties, des mécanismes d'arbitrage sont prévus. Des dispositifs de sauvegarde (exemple les otages) peuvent être utilisés pour s’assurer de la bonne exécution contractuel et prévenir tout comportement opportuniste. En phase d'exécution: les instruments de contrôle servent à assurer une supervision directe fréquente du partenaire. L’évaluation et la rémunération s’effectuent sur la base de la qualité des résultats et/ou des comportements spécifiés à l'avance dans le contrat. Le suivi complet et détaillé qui s’appuie sur des règles et procédures nécessite un transfert d’informations régulier entre les parties. Le système d'informations inter organisationnelles est ainsi un dispositif indispensable à la mise en œuvre de ce mode de contrôle (Nogatchewsky, 2009). Le tableau $n^{\circ} 1$ ci-dessous résume ces trois modes de gouvernance. Dans le cadre de cette recherche, nous retiendrons deux modèls de contrôles : les différents types de contrôle (évaluation du travail, suivi et audit, supervision directe, partage des informations, communication approfondie et fréquente, comportement) et les différents types de contrat de partenariat qui peuvent être à court ou à long terme.

Tableau 1: Les composants de l'enchevêtrement organisationnel

\begin{tabular}{|c|c|c|c|}
\hline $\begin{array}{c}\text { Formes } \\
\text { d'organisations }\end{array}$ & Marché & Réseau & Firme \\
\hline Coordination & $\begin{array}{c}\text { Concurrence par les } \\
\text { prix, ajustement par la } \\
\text { négociation, } \\
\text { formalisation par le } \\
\text { contrat. }\end{array}$ & $\begin{array}{c}\text { Coopération } \\
\text { réciproque, } \\
\text { ajustement par la } \\
\text { confiance, } \\
\text { formalisation par les } \\
\text { conventions. }\end{array}$ & $\begin{array}{c}\text { Intégration des } \\
\text { activités, ajustement } \\
\text { par l'autorité, } \\
\text { formalisation par les } \\
\text { règles. }\end{array}$ \\
\hline
\end{tabular}

Source : Baroncelli et Assens, 2002 p. 17

\section{La performance des réseaux de PME}

La performance de l'organisation demeure un enjeu central pour la recherche en gestion. Ce terme est largement utilisé sans que sa définition ne fasse l'unanimité. Pour Lebas (1995) il s'agit d'un concept difficile à définir, pour lequel quelques auteurs s'accordent sur les variables qui permettent de l'appréhender. Ce fait reflète la polysémie du mot. Elle s’apprécie souvent en termes d'efficacité et d'efficience. L'efficacité traduit la capacité à atteindre ses objectifs, tandis que l'efficience renvoie plutôt à l'aptitude à maximiser le rapport entre les résultats obtenus et les moyens engagés. La rentabilité financière ou la productivité du travail par exemple, sont des indicateurs d'efficience. L’efficacité apparait comme une notion plus large, et donc plus complexe que l'efficience qui elle, peut se mesurer par un ratio. Les objectifs 
peuvent représenter les finalités très larges, englobant un horizon temporel lointain ou répondre à une demande sociale élargie. La notion de performance a profondément évolué avec l'apparition et le développement de nouvelles formes d'organisation de l'activité économique. Les éléments qui permettent de définir la performance de ces réseaux d'entreprises sont jusqu'ici trop variables d'un contexte à l'autre et mal connus. Cette mauvaise connaissance se traduit par le fort taux de mortalité de ces entreprises en coopération (ou en réseau). La performance demeure toutefois relative à un contexte choisi en fonction de la stratégie (Lebas 1995). Et les critères retenus pour mesurer l'efficacité sont divers. Alors que Kalika (1988) regroupe les indicateurs de performance autour de trois dimensions dominantes : économique, organisationnelle et sociale, Morin et al., (1996), dans leur modèle quadridimensionnel, identifient les dimensions économique, sociale, systémique et politique du concept de performance. Cette dernière dimension évalue la satisfaction des partenaires extérieurs. Aujourd'hui apparaissent des critères éthiques qui permettent aux entreprises d'évaluer leurs pratiques sur des valeurs telles que la citoyenneté, l'environnement ou l'impact local. Pour Capiez (2008), ces dimensions sont multiples et peuvent être regroupées en deux catégories essentielles, une dimension objective à la fois économique (efficience) et systémique (pérennité de l'organisation), et une dimension subjective à la fois sociale (valeur des ressources humaines) et sociétale (légitimité de l'organisation). Pour compléter la mesure financière (efficience) privilégiée au départ, le contrôle de gestion propose une approche plus synthétique, comme celle du «Balanced Scorecard » (Kaplan et Norton, 1992) organisé en quatre dimensions: financière, clients, processus internes de gestion, apprentissage et croissance, avec des indicateurs aussi bien physiques et qualitatifs que financiers. En matière d'évaluation des réseaux, Heitz et Douard (2000) proposent une grille d'évaluation fondée sur les objectifs et stratégies des partenaires, les actifs du réseau, les risques perçus, le niveau d'implication de chaque partenaire, la logique de fonctionnement (additivité ou intégration), l'équilibre de coopération et l'exploitation des produits fournis par le réseau, avec des indicateurs stratégiques, de contrôle de gestion et de comptabilité (Capiez, 2008). Les mesures économiques et financières (par exemple, rentabilité des actifs et rentabilité des fonds propres), concurrentielles (par exemple, rentabilité commerciale et part de marché), boursières (par exemple, rentabilités anormales), de satisfaction des partenaires, de survie font partie de l'ensemble des variables destinées à évaluer la performance des réseaux d'entreprises (Geringer et Hebert, 1991). Quand à Powell et al. (1996), ils retiennent le taux de croissance de l'entreprise comme mesure de performance des entreprises en collaboration. 
Dans le cas d'études portant sur la collaboration entre entreprises de haute technologie, l'indicateur de performance généralement utilisé est le degré d’innovation (Thouraya et al., .2006). En général, deux types de mesures à la fois objectives et subjectives sont associées. Par exemple, Mohr et Speakman (1994) définissent deux indicateurs du succès du partenariat : l'indicateur objectif concerne le volume des échanges entre partenaires ; l'indicateur subjectif est la satisfaction des deux partenaires. En fait un réseau est réussi à partir du moment où il réalise ses objectifs. Les critères financiers tels la rentabilité des capitaux investis et la croissance du profit présentent deux limites : ils ne traduisent pas forcement la totalité et l'étendus des objectifs poursuivis (on peut avoir un résultat défavorable alors que la firme progresse, améliore sa position concurrentielle et l'apprentissage), lorsqu'on aborde les réseaux pour lesquels il n’y a pas création d’une entité indépendante, il n'existe pas de base commune sur laquelle on puisse faire reposer le calcul des indicateurs. Cela dit, Gulati (1998), met en garde par rapport à l'utilisation d'indicateurs financiers et comptables à cette fin. Selon eux, les rendements financiers sont obtenus par d'autres mécanismes et ne reflètent pas uniquement la valeur ajoutée de la collaboration. Dès lors, il nous semble que la notion de degré de satisfaction de chaque partie par rapport à la relation apparait comme la mesure la plus pertinente pour évaluer le succès d'une relation de partenariat (Brulhart, 2002) ${ }^{51}$; nous y avons ajouté la réduction des coûts. La tendance récente de la littérature met moins l'accent sur des mesures objectives et davantage sur la perception de la performance par les dirigeants.

\section{La relation entre le contrôle et la performance des réseaux de PME dans l'approche transactionnelle}

« Les études sur le succès des réseaux d’entreprises réalisées dans la dernière décennie indiquent que l'évaluation de la performance est un facteur de succès essentiel. Toutefois, un grand nombre de travaux sur la performance des réseaux se concentrent sur l'influence de l'environnement, le degré d'interaction des partenaires, les caractéristiques des partenaires ou des caractéristiques organisationnelles sur la performance » (Bandeira et al., 2011) ; malgré l’importance de ces recherchent, très peu se sont intéressées à

\footnotetext{
${ }^{51}$ Etoundi suite à sa communication au CAFRAD p.11 sur la contribution à la connaissance du mode de création et de fonctionnement des accords de coopération entre PME francophones arrive à la conclusion que « Cela devrait éviter de conclure hâtivement que les accords de coopération cessent du fait d'un échec nécessairement. Bien au contraire il souligner que la durabilité d'un accord de coopération n'est pas une réussite car il est normal que les partenaires se séparent lorsqu'ils ont atteint leurs objectifs. En outre, on pourrait utilement relever que la notion de succès est elle-même ambiguë parce qu'on ne sait jamais si l'on parle de réussite du commun ou des avantages stratégiques tirés de l'accord de coopération par tel autre partie ».
} 
l'influence du contrôle formel sur la performance. Nous n'en relevons aucune dans le contexte camerounais.

Pour Dupuy et Guibert (1997), au début d'une relation clientfournisseur, une formalisation contractuelle peut rassurer les partenaires et permettre de créer une vision partagée des performances à accomplir. Lorsque la relation est établie et stabilisée, la formalisation du contrôle n'a pas d'influence sur le climat de confiance; mais le suivi technologique systématique crée plutôt la défiance.

Nous pouvons rappeler que les auteurs issus du courant stratégique d'analyses des réseaux d'entreprises ont souligné l'importance du contrat (Miles et Snow, 1986), ou plus globalement des arrangements institutionnels pour le management et le contrôle des configurations complexes (Ring et Van de van, 1992 et 1994). Dans leur définition du réseau dynamique, Miles et Snow (1986) recommandent le choix du contrat comme mode essentiel de contrôle, associé à une rémunération en fonction des résultats et une diffusion totale de l'information entre les membres.

Delerue (2004) réalise une étude sur la performance des alliances de PME (gestion duale du risque relationnel). Celle-ci a été menée auprès d'une vingtaine de dirigeants de PME du secteur de la biotechnologie ayant au moins une alliance avec une autre entreprise privée. La motivation dans le choix des firmes était d'avoir un échantillon suffisamment varié en termes de structure des accords et de caractéristiques du partenaire de telle sorte que les managers soient exposés à des environnements différents. Elle fait la proposition suivante : Il existe probablement un lien négatif entre la performance de l'alliance et les instruments de contrôle autonomes formels. Elle aboutit à la conclusion suivante : le contrat n'influence pas la perception des risques et en ce sens, n’apparaît pas être un instrument de contrôle pertinent. L'absence de performance est attribuée principalement à l'opportunisme, à l'influence excessive du partenaire, et aux problèmes d'incompréhension. Selon cette proposition, la manière dont les dirigeants gèrent les risques a une influence sur la manière dont ils perçoivent la performance de l'accord. Après l'examen de ces travaux nous nous positionnons dans l'optique d'une relation positive entre le contrôle formel et la performance. Nous formulons les hypothèses suivantes :

\section{Hypothèse 1 : la mise en place des différents types de contrôle par la PME améliore sa performance.}

Le recours à des contrats de long terme permet de minimiser les coûts de transaction. Le contrat à court terme, de type « résultat », peut être utile lorsque la coopération porte sur un projet précis ; néanmoins, un contrat à long terme entraîne des perspectives de développement en commun 
(Dumoulin, 1997). Il implique que les parties apprennent ensemble à coopérer et développent l'apprentissage entre les membres.

\section{Hypothèse 2 : les différents types de contrat ont une influence positive sur le niveau de performance des PME en réseaux.}

Hypothèse 2 a : les contrats de moins de 6 mois ont une influence positive sur le niveau de performance des partenaires impliqués dans le réseau.

Hypothèse $2 \mathrm{~b}$ : les contrats entre 6 mois et deux ans ont une influence positive sur le niveau de performance des partenaires impliqués dans le réseau.

Hypothèse 2 c : les contrats de plus de deux ans ont une influence positive sur le niveau de performance des partenaires impliqués dans le réseau.

Hypothèse $2 \mathrm{~d}$ : l'existence de plusieurs types de contrats a une influence positive sur le niveau de performance des partenaires impliqués dans le réseau.

\section{Méthodologie de l'étude}

Pour tester ces hypothèses les informations sont collectées auprès d'un échantillon de PME camerounaises fonctionnant avec leurs partenaires ${ }^{52}$ sous forme de réseau. Les réseaux peuvent être étudiés de deux façons principales : soit par une approche inter organisationnelle, à partir de l'analyse de plusieurs études de cas représentatifs, soit par une approche intra-organisationnelle, en se plaçant du point de vue de l'entreprise insérée dans un réseau (Capiez 2008). C'est la deuxième approche que nous avons privilégiée car elle permet d'interroger un plus grand nombre d'entreprises (nous analysons la PME insérée dans le réseau et non la performance du réseau, que la relation soit verticale ou horizontale, la perception devrait être identique). Nous avons choisi la méthode non probabiliste pour laquelle l'erreur d'échantillonnage reste inconnue, la procédure consistant à utiliser le jugement pour identifier un échantillon représentatif. Parmi les méthodes non probabilistes nous avons adopté la méthode par quota qui nous a semblé plus adaptée à notre recherche. La recherche ayant une approche en termes de compétences et de ressources, nous nous sommes intéressés aux PME des secteurs manufacturiers et logistiques. Pour l'ONUDI, les PME manufacturières peuvent jouer un rôle clé dans l'impulsion et la durabilité de la croissance économique et le développement équitable dans les pays en voie de développement. Prises

${ }^{52}$ Les partenaires pouvant être des fournisseurs, des clients, des concurrents avec qui l'entreprise entretien des relations stratégiques à long terme. Dans un réseau on parle de partenaire stratégique. 
individuellement les PME sont incapables de saisir les opportunités de marché que requiert la production d'importante quantités, des normes homogènes et un approvisionnement régulier. Les données sont récoltées à partir d'un questionnaire que nous avons adopté après une étude exploratoire auprès des dirigeants de ces PME. Il comporte deux thèmes: un sur le contrôle dans l'approche transactionnelle et l'autre sur la performance des PME évoluant dans les relations de réseau.

Nous avons comme variable expliquée la performance ; cette variable est mesurée à partir des items issus de la littérature sur des échelles de cinq point Likert (allant de pas du tout d'accord à tout à fait d'accord). Egalement, les échelles de Canon et Perrault (1999) ont été combinées aux échelles proposées par Ganesan (1994), Stanket et al. (1996) et par Blankernburg et al. (1999). La série de question portant sur la notion perçue de succès ou d'échec de l'implication dans le réseau par l'entreprise a été a été appréhendée à partir de deux types de mesures à la fois objectives et subjectives. Les mesures « objectives » de l'accroissement de la performance sont contenues dans trois questions : une relative à la variation des coûts, une autre relative au développement de la qualité du service, et au final une relative à la réduction de l'actif circulant et immobilisé (Dumoulin et al, 2000). Nous avons ajouté une quatrième question après une étude exploratoire l'augmentation de chiffre d'affaires. La mesure subjective étant contenue dans une question relative à la satisfaction globale de s’être engagé dans le réseau. Nous avons donc onze items pour les questions sur la performance. Pour les variables expliquées nous avons d'une part les différents types de contrôle: l'évaluation du travail, le suivi et l'audit, le partage de l'information, la communication profonde et approfondies, le résultat, le comportement et la supervision directe. Et d'autre part les différents types de contrats (le contrat à court terme d'une durée de moins de six mois; le contrat à moyen terme d'une durée de de six mois à deux années; le contrat à long terme d’une durée de plus de deux années ; plusieurs contrat différenciés par type de partenaire).

\section{L’échantillon}

La population de notre étude porte exclusivement sur les PME camerounaises dont l'effectif du personnel employé est inférieur à cent et le chiffre d'affaires annuel est inferieur ou égal à un milliard. L’échantillon est constitué des PME du domaine logistique et de PMI manufacturières. Nous considérons que le secteur manufacturier est celui dont la fonction économique essentielle est la transformation selon un processus industriel des matières premières pour obtenir des produits tangibles soit pour la consommation finale, soit pour le consommateur industriel (Djeumene, 2004). Aussi, le secteur manufacturier est constitué des branches R-02 à R- 
24 de la nomenclature de la Direction de la Statistique et de la comptabilité Nationale du Cameroun (DSCN). Il s'agit ainsi des branches dont les entreprises sont généralement consultées au cours des enquêtes de conjoncture réalisée par la DSCN. Par ailleurs, bien qu'adoptant cette définition du secteur manufacturier, nous excluons de cet univers d'étude la branche R-24 constituée essentiellement des entreprises fournissant l'eau et l'énergie et connues pour être les grandes entreprises. Pour les PME du secteur logistique, nous les définissons comme celles dont l'objectif est l'optimisation des flux physiques constitués de mouvements et manutentions des matières, marchandises et produits depuis les sources d'approvisionnements jusqu'au point d'utilisation (Darbelet, 2004). Il est important de mentionner que les réponses au questionnaire peuvent être fournis aussi bien par le Directeur Général (DG), que par ses proches collaborateurs dûment mandatés à cet effet. En élargissant l'éventail des répondants, ce détour permet de réduire la probabilité de faire face aux non réponses du fait de l'emploi de temps surchargé des principaux responsables. Notre échantillon est constitué des PME implantées dans les villes de Douala, Yaoundé, les régions de l'Ouest, Sud Ouest et Nord Ouest car ces villes et régions regroupent environ $79 \%$ des PME du territoire camerounais. Par souci de précision statistique et de crédibilité d'une part, des politiques des entreprises et des contraintes financières qui peuvent influencer la taille de l'échantillon d'autre part, nous avons choisi d'interroger 100 PME. L'enquête a eu lieu entre les mois de Mars et Novembre 2012. Les tableaux 2 et 3 ci-dessous nous indiquent la répartition de ces PME par régions et les activités de ces PME. La répartition par ville ou régions se présentent comme suit :

Tableau 2 : retour des questionnaires et répartition de l'échantillon

\begin{tabular}{|c|c|c|c|}
\hline \multicolumn{2}{|c|}{ Information sur les 100 questionnaires administrés } & \multicolumn{2}{|c|}{$\begin{array}{l}\text { Répartition des questionnaires } \\
\text { par régions ou villes }\end{array}$} \\
\hline Retour des questionnaires & Nombre & Villes ou régions & Effectifs \\
\hline Questionnaires administrés : & 100 & Douala & 30 \\
\hline $\begin{array}{l}\text { Nombre d'enquêtés ayant refusé de } \\
\text { répondre au questionnaire sans raison : }\end{array}$ & 15 & Yaoundé & 15 \\
\hline $\begin{array}{c}\text { Nombre d’enquêtés ayant refusé de } \\
\text { répondre à cause des « questions } \\
\text { gênantes » : }\end{array}$ & 14 & Ouest & 7 \\
\hline Questionnaires à moitiés remplis : & 9 & Sud-ouest & 1 \\
\hline $\begin{array}{l}\text { Questionnaires abandonnés par nous } \\
\text { même suite aux Rendez-vous manqués : }\end{array}$ & 8 & Nord-ouest & 1 \\
\hline Questionnaires exploités : & 54 & Total & 54 \\
\hline
\end{tabular}

Source : obtenu à partir de notre collecte de données 
Tableau 3 : Activités principales des entreprises de l'échantillon

\begin{tabular}{|c|c|c|c|c|}
\hline \multicolumn{2}{|c|}{ Activités principales } & Fréquences & $\%$ & $\%$ cumulés \\
\hline 1 & Industrie caoutchouc et fabrication art plastique & 2 & 3,7 & 3,7 \\
\hline 2 & Boulangerie pâtissérie & 8 & 14,8 & 18,5 \\
\hline 3 & Conseil en management & 3 & 5,6 & 24,1 \\
\hline 4 & Impression et édition & 5 & 9,3 & 33,3 \\
\hline 5 & Transformation des produits d'origines agricoles & 2 & 3,7 & 37 \\
\hline 6 & Fabrication des boissons & 7 & 13 & 50 \\
\hline 7 & Approvisionnement & 4 & 7,4 & 57,4 \\
\hline 8 & Autres industries alimentaires & 13 & 24,1 & 81,5 \\
\hline 9 & Fabrication matériaux & 4 & 7,4 & 88,9 \\
\hline 10 & Industrie papier articles en papiers & 2 & 3,7 & 92,6 \\
\hline 11 & Industrie manufacturières diverses & 2 & 3,7 & 96,3 \\
\hline 12 & Fabrication d'appareils mécaniques électriques et & 2 & 3,7 & 100 \\
& métalliques & & & \\
\hline & Total & 54 & 100 & \\
\hline
\end{tabular}

Source : obtenu à partir de notre collecte de données

\section{Méthode d'analyse}

Pour le traitement des données nous avons utilisé le logiciel SPSS (V10) qui nous permettra de faire des analyses uni variées, bi variées et multi variées (analyses descriptives). L'analyse factorielle et l'analyse factorielle de correspondance permet de vérifier les échelles de mesure. Concrètement, nous avons d'abord réalisé une ACP sur l'ensemble des items constitutifs des variables exogènes (le contrôle dans l'approche transactionnelle); puis sur l'ensemble des items de la performance afin d'éviter tout risque de multicollinéalité. Nous avons testé l'unidimensionnalité des facteurs ainsi identifiés en renouvelant sur chacun d'entre eux une nouvelle ACP. A chaque fois nous avons observé la répartition des items sur les facteurs avant et après une rotation de Varimax. Les critères proposés par (Evrard et al 1997) ont été retenus pour purifier les échelles puisque nous avons éliminé les items n'ayant aucune contribution factorielle supérieure ou égale à 0,50 sur l'une des composantes principales identifiées. Après avoir étudié la validité des échelles, nous nous sommes penchés sur la fiabilité des facteurs issus de l'analyse factorielle en utilisant la mesure de l'alpha de Crombach. Ainsi, quand la valeur de l'alpha est proche de 1, l'échelle a une bonne cohérence interne et les questions sensées mesurer la même chose mesurent effectivement la même chose. La régression et le calcul du coefficient de corrélation nous permettront de voir s'il y a relation entre variables explicatives et variable expliquée. 


\section{Résultats et discussions Résultats}

Le concept de performance est mesuré par onze items ; parmi ces onze items nous avons deux items renversés, il s’agit des questions Q36 et Q38. Nous avons réalisé le test de pertinence de l'analyse en composante principales dit test de KMO qui donne la valeur 0,583 considéré comme bon ainsi que le test de Bartlett qui est de 275,799 avec un seuil de signification très faible $(0,000)$, permettant d'accepter les résultats de cette analyse factorielle. La matrice de composante après rotation se trouve dans le tableau ci-après :

Tableau 4 : analyse des items relatives à la performance

\begin{tabular}{|c|c|c|c|c|}
\hline & \multicolumn{4}{|c|}{ Composantes } \\
\hline & 1 & 2 & 3 & 4 \\
\hline $\begin{array}{l}\text { Globalement nous sommes satisfaits de cette association. } \\
\qquad \mathrm{r}(\mathrm{Q} 36)\end{array}$ & & & 0,70 & \\
\hline $\begin{array}{c}\text { Nous sommes très mécontent des résultats de cette } \\
\text { association. (Q37) }\end{array}$ & & $\begin{array}{c}- \\
0,762 \\
\end{array}$ & & \\
\hline $\begin{array}{l}\text { L’association de notre entreprise avec ce partenaire est un } \\
\text { succès. } r(Q 38)\end{array}$ & & & & 0,866 \\
\hline $\begin{array}{c}\text { D’une façon générale, nous dirons que les résultats de } \\
\text { notre association avec ces partenaires ont largement } \\
\text { dépassé nos attentes. (Q39) }\end{array}$ & & & & 0,633 \\
\hline $\begin{array}{c}\text { Si nous devions procéder à une évaluation de la } \\
\text { performance de notre association avec ces partenaires, elle } \\
\text { serait très exceptionnelle. (Q40) }\end{array}$ & 0,597 & & & \\
\hline $\begin{array}{c}\text { La relation de partenariat avec ces partenaires est très } \\
\text { profitable. (Q41) }\end{array}$ & 0,806 & & & \\
\hline Cette coopération permet de réduire les coûts. (Q42) & 0,662 & & & \\
\hline $\begin{array}{c}\text { Cette coopération améliore la qualité du service } \\
\text { (production). (Q43) }\end{array}$ & 0,895 & & & \\
\hline $\begin{array}{c}\text { Cette coopération entraine une réduction de l'actif circulant } \\
\text { et des immobilisations. (Q44) }\end{array}$ & & & 0,817 & \\
\hline Cette coopération améliore notre chiffre d’affaires. (Q45) & & 0,648 & & \\
\hline $\begin{array}{l}\text { Cette coopération nous permet d’être plus compétitifs. } \\
\text { (Q46) }\end{array}$ & 0,902 & & & \\
\hline
\end{tabular}

Méthode d'extraction : analyse en composantes principales

Méthode de rotation : varimax avec normalisation de Kaiser

Source : logiciel de traitement des données

Nous avons quatre facteurs dont le premier représente 36,28 \% des variances et les deuxième, troisième quatrième facteurs représentent respectivement $17,19 \%, 12,40 \%$ et $9,34 \%$ des variances : ce qui est faible. Nous constatons que ce sont les questions renversées qui se trouvent sur le deuxième, troisième et quatrième facteurs, nous les supprimons et en renouvelant l'analyse, nous avons cette fois un KMO de 0,827 et le test de Bartlett de 131,158 et une signification de (0,000), ce qui nous permet une 
fois de plus d'accepter les résultats de l’analyse factorielle. La matrice des composantes devient :

Tableau 5 : facteur retenu pour le concept performance

\begin{tabular}{|c|c|c|}
\hline $\begin{array}{c}\text { Si nous devions procéder à une évaluation de la performance de } \\
\text { notre association avec ces partenaires, elle serait très } \\
\text { exceptionnelle. (Q40) }\end{array}$ & 0,644 & 0,415 \\
\hline $\begin{array}{c}\text { La relation de partenariat avec ces partenaires est très profitable. } \\
\text { (Q41) }\end{array}$ & 0,852 & 0,726 \\
\hline Cette coopération permet de réduire les coûts. (Q42) & 0,754 & 0,568 \\
\hline $\begin{array}{c}\text { Cette coopération améliore la qualité du service (production). } \\
\text { (Q43) }\end{array}$ & 0,911 & 0,830 \\
\hline Cette coopération nous permet d'être plus compétitifs. (Q46) & 0,851 & 0,725 \\
\hline Valeurs propres & $\mathbf{3 , 2 6 4}$ & \\
\hline \% de variance expliquée & $\mathbf{6 5 , 2 8 4}$ & \\
\hline Coefficient d'alpha de crombach & $\mathbf{0 , 8 5}$ & \\
\hline
\end{tabular}

Source : logiciel de traitement des données

Cette dernière solution unidimensionnelle permet d'expliquer 65, 284 $\%$ de la variance ; les communalités et les composantes de chaque item sont respectivement supérieures à 0,40. La matrice des composantes indique que tous les items ont un coefficient structurel supérieur à 0,64. Les items Q40, Q41, Q42, Q43, Q46, sont donc conservés pour la suite de l'analyse. Pour mesurer la cohérence interne de ces items, nous avons recherché son coefficient d'alpha de crombach qui est de 0,85 qui doit être considéré comme très satisfaisant parce qu'il est supérieur à la valeur minimale recommandée $(0,60)$. Un rapprochement avec les résultats de (Brulhart 2002) nous permet de faire les remarques suivantes : dans un premier temps nous avons retenu les items que lui pour mesurer la performance du partenariat, ensuite nous avons ajouté quatre items de (Dumoulin et al, 2000) pour mesurer la performance ; et enfin nous avons ajouté un item Q46. Sur les six items de (Brulhart, 2002), deux ont été retenus, et sur les quatre deuxièmes items du deuxième auteur ci-dessus deux ont été retenus en plus du dernier issu de notre propre analyse. Le coefficient d'alpha de crombach étant de 0,92 chez (Brulhart, 2002), nous avons jugé le notre $(0,85)$ acceptable.

Le concept des différents types de contrôle : ce concept est mesuré avec sept items. Nous avons réalisé le test de pertinence de l'analyse en composantes principales dit test de KMO qui donne la valeur 0,558, considérée comme bonne ainsi que le test de Bartlett qui est de 96,476 avec un seuil de signification très faible $(0,000)$, permettant d'accepter les résultats de cette analyse factorielle. La matrice de composante après rotation se trouve dans le tableau ci-après : 
Tableau 6 : L'analyse des items relatifs aux différents types de contrôle

\begin{tabular}{|c|c|c|c|}
\hline & \multicolumn{3}{|c|}{ Composantes } \\
\cline { 2 - 4 } & 1 & 2 & 3 \\
\hline Evaluation du travail & 0,658 & & \\
\hline Suivi et audit & & 0,706 & \\
\hline Supervision directe & & & 0,947 \\
\hline Partage des informations & 0,876 & & \\
\hline Communication approfondies et fréquentes & 0,816 & & \\
\hline Comportement & & 0,856 & \\
\hline Résultat & & 0,169 & \\
\hline
\end{tabular}

Méthode d'extraction : analyse en composantes principales.

Méthode de rotation : varimax avec normalisation de Kaiser. Source : SPSS (V10).

Nous constatons trois facteurs dont le premier représente 35,062 \% des variances, les deuxième, et troisième facteurs représentent respectivement 20, $913 \%$, et $17,397 \%$ des variances : ce qui est faible. On déduit que le résultat a un coefficient structurel faible, et une dispersion entre le deuxième et le troisième facteur du comportement, suivi et audit et supervision directe ; nous les supprimons et nous arrivons à un KMO de 0,631 et le test de Bartlett de 40,143 avec une signification de $(0,000)$, nous permettant une fois de plus de valider les résultats de l'analyse factorielle. La matrice des composantes devient :

Tableau 7 : Le facteur retenu pour le concept types de contrôle

\begin{tabular}{|c|c|c|}
\hline Items & F1 & Commnalités \\
\hline Evaluation du travail & 0,723 & 0,522 \\
\hline Partage des informations & 0,881 & 0,776 \\
\hline Communication approfondies et fréquentes & 0,832 & 0,692 \\
\hline Valeurs propres & 1,991 & \\
\hline \% de variance expliquée & 66,35 & \\
\hline Coefficient d'alpha de crombach & 0,73 & \\
\hline
\end{tabular}

Source : SPSS (V10).

Cette dernière solution multidimensionnelle permet d'expliquer 66, $35 \%$ de la variance ; les communalités et les composantes de chaque item sont respectivement supérieures à 0,50. La matrice des composantes indique que tous les items ont un coefficient structurel supérieur à 0,65. Les items : évaluation du travail, partage des informations, communications approfondies et fréquentes sont conservés pour la suite de l'analyse. Pour mesurer la cohérence interne de ces items, nous avons abouti à un coefficient alpha de crombach de 0,73 considéré comme satisfaisant, car supérieur à la valeur minimale recommandée $(0,60)$. Ces items sont ainsi retenus pour la première fois pour les tests d'hypothèses. Et pour ce faire nous avons réalisé les tests de corrélation de Pearson et la régression linéaire. 


\section{Hypothèse 1 : la mise en place des différents types de contrôle par la PME améliore sa performance.}

Tableau 8 : La corrélation de Pearson de l'hypothèse 1

\begin{tabular}{|cc|c|}
\hline & & Performance \\
\hline Différents type de contrôle & corrélation Pearson & 0,130 (NS) \\
& Sig (bilatérale) & 0,348 \\
$\mathrm{~N}$ & 54 \\
\hline
\end{tabular}

(NS) Non significatif

Source : SPSS (V10).

Nous observons à partir de ce tableau qu'il n'existe pas une relation bilatérale entre les différents types de contrôle et la performance des entreprises engagées dans les réseaux. La régression n’était plus nécessaire ici pour percevoir le sens de la relation.

\section{Hypothèse 2 : les différents types de contrat ont une influence positive sur le niveau de performance des PME en réseaux.}

Hypothèse $2 \mathrm{a}$ : les contrats de moins de 6 mois ont une influence positive sur le niveau de performance des partenaires impliqués dans le réseau.

Hypothèse $2 \mathrm{~b}$ : les contrats entre 6 mois et deux ans ont une influence positive sur le niveau de performance des partenaires impliqués dans le réseau.

Hypothèse $2 \mathrm{c}$ : les contrats de plus de deux ans ont une influence positive sur le niveau de performance des partenaires impliqués dans le réseau.

Hypothèse $2 \mathrm{~d}$ : l'existence de plusieurs types de contrats a une influence positive sur le niveau de performance des partenaires impliqués dans le réseau.

Le test de corrélation nous donne le tableau ci-dessous :

Tableau 9 : La corrélation de Pearson des hypothèses 2 a, 2 b, 2 c, et 2 d

\begin{tabular}{|cc|c|}
\hline & & Performance \\
\hline Le contrat de moins de 6 mois & corrélation Pearson & $-0,349^{* *}$ \\
& Sig (bilatérale) & 0,010 \\
& $\mathrm{~N}$ & 54 \\
\hline Les contrats entre 6 mois et 2 ans & corrélation Pearson & $-0,057$ (NS) \\
& Sig (bilatérale) & 0,680 \\
& $\mathrm{~N}$ & 54 \\
\hline Les contrats de plus 2 ans & corrélation Pearson & 0,121 (NS) \\
& Sig (bilatérale) & 0,384 \\
& $\mathrm{~N}$ & 54 \\
\hline L'existence de plusieurs types de contrats & corrélation Pearson & $-0,077$ (NS) \\
& Sig (bilatérale) & 0,582 \\
& $\mathrm{~N}$ & 54 \\
\hline
\end{tabular}

**la corrélation est significative au seuil de 0,01 (bilatérale)

(NS) Non significatif

Source : SPSS (V10). 
A partir de ce tableau, nous observons d'une part qu'il existe une relation bilatérale entre les contrats à moins de 6 mois et la performance des entreprises engagées dans les réseaux; d’autre part qu’il n’existe pas de relation entre les autres formes de contrats et la performance. La relation entre les contrats à moins de 6 mois et la performance est moyenne : son coefficient de corrélation est de -0,349. Cependant, ne percevant pas encore le sens de cette relation, la régression linéaire nous permettra de répondre à cette interrogation.

Le modèle obtenu est significatif $(p<0,05)$, le lien entre les contrats de moins de 6 mois et la performance est significatif $(\mathrm{t}=-0,2683<-2)$ et positif (R2 ou coefficient standardisé de 0,105). Ceci nous permet de dire que les contrats de moins de 6 mois influencent négativement la performance.

\section{Discussion et conclusion}

Les différents types de contrôle n’ont pas une influence positive sur le niveau de performance des entreprises du réseau. L'hypothèse 1 n'est pas validée. Ces différents types de contrôle ont été opérationnalisés par l'évaluation du travail, le partage des informations, le suivi et l'audit, la supervision directe, le résultat et le comportement. Ce résultat se rapproche de celui de Delerue (2004) qui n’établit pas une relation entre les différents types de contrôle et la performance du partenaire dans le réseau.

Des hypothèses 2 a, 2 b, 2 c, et 2 d, seule 2 a est validée. Le contrat à long terme qui est généralement décrit comme élément de confiance n’a pas été validé dans notre contexte comme étant explicatif de la performance. Nous avons plutôt les contrats de moins de six mois qui influencent négativement la performance. Ce résultat se rapproche des recherches de Torres (1999) pour qui «L’insécurité contractuelle, légale et judiciaire dans les pays en voie de développement oblige l'entrepreneur à créer un groupe ethniquement homogène et des réseaux d’échanges personnalisés en vue de réduire le coût inhérent au non respect des contrats » p. 11. Ce recourt important aux contrats de moins de six mois peut être dû au non respect des termes du contrat par les partenaires, à l'insécurité légale et judiciaire qui amène les partenaires à évaluer d'abord l'exécution d'un contrat de moins de six mois, avant de passer à un autre contrat.

En ce qui concerne la performance, nos résultats se rapprochent des conclusions de Geyskens et al. (1999) selon lesquelles la satisfaction est un état affectif résultant de l'appréciation de tous les aspects d'une relation de travail d'une entreprise avec une autre entreprise. Ainsi, pour cet auteur la satisfaction peut être considérée comme l'évaluation affective globale portée sur la relation de partenariat. En ce sens, la satisfaction permet d'évaluer l'atteinte des objectifs et le caractère pertinent et productif de la 
relation. En outre, la satisfaction apparaît comme un concept riche qui offre la possibilité d'être décliné en plusieurs catégories portant respectivement sur les différents domaines de la relation (satisfaction par rapport à l'atteinte des objectifs en général, aux compétences du partenaire, au profit dégagé par le partenariat, au rapport coût / bénéfice...).

Le contrat est nécessaire à l'organisation des rapports entre les partenaires d'un réseau. Mais il est incomplet par nature, au sens des économistes. Il ne peut seul répondre à la question de leur coordination : le détail excessif du futur envisageable de la relation ests par essence inefficace, voire même dangereux puisqu'impliquant des risques de blocage et faisant croire naïvement à une bonne protection en cas de problème. Cette incomplétude, loin d'être un handicap, constitue un atout dans la mesure ou elle permet d'autres modes de régulations, la confiance entre autre. Cette incomplétude est un facteur de flexibilité qui permet la reconfiguration du contrôle formel indispensable au fonctionnement du réseau.

\section{References:}

1. Bandeira L. K. R. et Retour D. (2011), «L'analyse de la performance des réseaux : une étude qualitative » IFBAE France pp. 470-484.

2. Baroncelli A. et Assens C. (2002), Réseau - Hiérarchie - Marché, AIMS.

3. Butera F, (1991), La métaphore de l'organisation : du château au réseau, Les éditions d'organisation, Paris.

4. Brulhart F. (2002), "le rôle de la confiance dans le succès des partenariats verticaux logistiques: le cas des coopérations entre industriels-alimentaires et prestataires logistiques » Revue finance contrôle comptabilité vol. 5, n4, pp.51-77.

5. Capiez A. (2008), «Réseau d'entreprise et performance: une approche empirique». Revue Comptabilité Contrôle Audit et institution(s)Tunis. $\quad$ http://halshs.archives-ouvertes.fr/halshs00548094, Soumis le : Samedi 18 Décembre 2010, vol.16, pp. 29:37.

6. Crottet S. (2001), Stratégies internationales en marketing des services le cas des petites et moyennes entreprises Suisse, Thèse présentée à la Faculté des sciences économiques et sociales de l’Université de Fribourg (Suisse).

7. Darbelet M. (2004), Economie d'entreprise, $2^{\text {ème }}$ édition, Foucher, Paris.

8. Delerue H. (2004), «Performance des alliances : une gestion duale du risque relationnel ", 13ème conférence de l'AIMS, Normandie, Vallée de seine, 2, 3 et 4 juin. 
9. Djeumene P. (2004), Propension à nouer des accords de coopération et compétitivité de PMI manufacturières camerounaises, Thèse de Doctorat Ph.D en Sciences de Gestion Université de Ngaoundéré.

10. Dumoulin R. (1997), « Marché, bureaucratie et clans dans les réseaux stratégiques », Comptabilité Contrôle Audit, tome 3, Vol. 2, pp. 4558.

11. Dumoulin G, Meschi P. x, Uhlig T. (2000), « Management, contrôle et performance des réseaux d'entreprises : étude empirique de 55 réseaux d'alliance » Rrevue finance contrôle comptabilité vol 3, $\mathrm{n}^{\circ} 222$, pp 81-112.

12. Dupuy et Guibert (1997), « La complémentarité entre le contrôle « formel » et contrôle « informel »: le cas de la relation clientfournisseur », Comptabilité Contrôle Audit, Tome 3 Vol 1, mars, pp. 39-52.

13. Evrard Y., Pras B., et Roux E. (2003), Market : étude et recherché en marketing, Nathan Paris.

14. Geyskens I. Steenkamp J. B. et Kumar N. (1999), «A Meta-analysis of Satisfaction in Marketing Channel Relationships ». Journal of Marketing Research, vol. 36, pp. 223-238.

15. Geringer J. et Herbert L. (1991), "Measuring performance of international joint venture", Journal of international business studies, vol 22, pp 249-264.

16. Gulati R. (1998), “Alliances and Networks”, Strategic Management Journal, vol 19, ${ }^{\circ} 2$, pp 293-317.

17. Guibert N. (1999), "La confiance en marketing : fondements et applications ", Recherche et Applications en Marketing, vol 14, $\mathrm{n}^{\circ} 1$, pp 1-19.

18. Gurviez P. et Korchia M. (2002), « Proposition d’une échelle de mesure multidimensionnelle de la confiance dans la marque", Recherche et Applications en Marketing, vol 17, ${ }^{\circ} 3$, pp 41-59.

19. Hammami A. et al, (2001), "Contribution à la conception et au pilotage d'une entreprise en réseau », $3^{\text {ème }}$ conférence MOSIM, du 25 au 27 avril Troyes France.

20. Harrigan K. R. (1985), "Strategic Alliances: the new role in global competition", The Columbian journal of Word Business, Vol. 22, $n^{\circ} 2$.

21. Heitz M. et Douard J. P. (2000), "Les coopérations inter entreprises : une grille de lecture des différentes formes et des évolutions". Revue Finance Contrôle Stratégie, vol. 3, $\mathrm{n}^{\circ}$. 4. pp 57-81.

22. Hasrouri L. (2007), " une synthèse des travaux sur le contrôle des RIO en France : état des lieux et voies de recherche ", association francophone de comptabilité, Poitier. 
23. Kalika M. (1988), Structures d'entreprises :réalités, déterminants, performances, Economica.

24. Kaplan R. S. et Norton D. P. (1992), "The Balanced Scorecard. Measures that Drive Performance ". Harvard Business Review, January-February, pp. 481-510.

25. Lebas M. (1995), «Le concept de performance », Travail, $n^{\circ} 34$, Printemps-Eté, pp.137-149.

26. Miles R. E. et Snow C. C. (1986), "Organizations: New Forms for New Concepts", California Management Review, vol. 28, n¹.

27. Mohr J., Speakman R. (1994), "Characteristics of Partnership Success, Partnership Attributes, Communication Behavior, and Conflict Resolution Techniques”, Strategic Management Journal, vol 15, pp 45-64.

28. Morin E. et al, (1996), " Mesurer la performance de l'entreprise », Revue Gestion, vol 21, $\mathrm{n}^{\circ}$ 3, pp 61-66.

29. Ngok evina J. F. (2008), « Gestion des ressources humaines dans les entreprises africaines : cas du Cameroun ».

30. Nogatchewsky G. (2009), "Contrôle inter organisationnel ", encyclopédie de comptabilité contrôle et audit pp. 625-633.

31. Podolny J.M. et Page K. (1998), "Network Forms of Organizations", Annual Review of Sociology, vol 24, pp 57-76.

32. Porter M. (1986), Competitive Advantage, Creating and Sustaining Superior Performance, The Free Press.

33. Powell W. W. Kenneth W. K. et Smith-Doerr L. (1996), "Interorganizational Collaboration and the Locus of Innovation: Networks of Learning in Biotechnology", Administrative Science Quarterly, Vol. 41, n. 1 pp. 116-145.

34. Quelin B. (2003), « Externalisation stratégique et partenariat de la firme patrimoniale à la firme contractuelle ", Revue Française de Gestion, ${ }^{\circ} 143$, pp. 13-26.

35. Ring P. S. et Van de Ven A. H. (1992), '”Structuring Cooperative Relationships Between Organizations". Strategic Management Journal, Vol.13, nº.

36. Ring P.S. Van de Ven A.H. (1994), "Developmental Processes of Cooperative" Interorganizational Relationships , Academy of Management Review, vol 19, $\mathrm{n}^{\circ} 1$, pp 90-118.

37. Roblédo C. (1997), "Contrôle et coentreprises internationales : la place du contrôle contractuel », Actes du colloque, Connivences d'acteurs, contrats, coopérations et métamorphose des organisations , Nancy-Luxembourg, mai, 658-680. 
38. Rouette F. (1999), « Le contrat de ' partenariat : là ou les parallèles se rencontrent ", Colloque de l'Institut de Formation en Gestion du Transport et de la Logistique, Montréal, 18 novembre.

39. Stank T. P. Daugherty P. J. et Ellinger A. E. (1996) "Information Exchange, Responsiveness and Logistics Provider performance”. The international journal of logistics management, Vol. 7, $\mathrm{n}^{\circ} 2$, pp.43-57.

40. Thouraya G.L. et al. (2006), «Influence de la collaboration sur la performance des PME hôtelières Suisse » 8ème CIFEPME.

41. Torres O. (1999), Les PMES, Dominos Flammarion.

42. Williamson O. E. (1975), Market and Hierarchies: analysis and antitrust implication, New York : The free Press. 